\title{
BIO.02 - Risk Assessment based approach to support the qualification of equipment: a case study application on a mammalian cell bioreaction system
}

\author{
Miguel Angel de la O Herrera ${ }^{1 *}$; Rodrigo Coelho Ventura Pinto ${ }^{1}$; Elezer Monte Blanco Lemes ${ }^{1}$; \\ Danilo Parmera ${ }^{1}$. \\ 1Fiocruz/Bio-Manguinhos.
}

Introduction: The equipment qualification within the pharmaceutical industry, besides being mandatory, is indispensable to verify that any equipment was designed, installed, and operates to perform the expected function. However, high technological complexity of innovative and customized systems makes it difficult to execute this task, requiring high human and financial resources. To face this situation, a risk analysis based approach has been used to simplify the qualification tests in key elements and critical functions within the system, resulting in the optimization of resources and reduction of qualification time, which in some cases may take years.

Objective: Optimize the qualification process of a Mammalian Cell Bioreaction System through the application of an FMEA-HAZOP Integrated Risk Assessment Methodology (FHRAM) that allows the identification of the most critical components and functions, the level of detailing, and amount of the tests needed to perform the qualification.

Methodology: First, through a multidisciplinary consensus, it was possible to define the approach, elements and procedure of application of theFHRAM. To identify the main operations and functions of the Bioreaction System, each component was analyzed and subsequently the FHRAM was applied. In the resulting risk report, actions and recommendations were proposed aiming to mitigate the risk level in the most critical elements. These elements will be used as basis to establish de qualification program of the Bioreaction System.

Results: Despite of the importance that each equipment has on the correct operation of the system, it was determined that the bioreactors hold the most critical functions and operations. Considering this, 9 critical functions were identified, being: (1) Supply of process gases; (2) Supply of solutions; (3) Process Gas Filtration; (4) Filtration of Exhaust Gases; (5) Heat Exchangers; (6) Medium Filtration; (7) CIP System; (8) Bioreactor tank; (9) Culture Transfer. In each group, it was observed that variations of process parameters like temperature, pressure and flow, represented the deviations classified as undesired, being with this characteristic approximately $75 \%$ of the total deviations, the remaining deviations were classified as acceptable, and no unacceptable deviations were identified. Finally, the capacity to detect failures allowed prioritizing treatment of deviations regardless of the risk level.

Conclusion: The importance of reducing the costs and resources necessary to perform equipment qualification is widely recognized within the pharmaceutical industry. After this work was concluded, it was possible to reduce considerably the amount of tests to verify the correct installation, operation and performance of the equipment by monitoring only the most critical functions. Furthermore, it was easily observed that by establishing procedures for instrument calibration and maintenance practices, the frequency of undesired events decreases considerably and in some cases even was completely eradicated. Finally, it was possible to establish response procedures in case of any potential failure is materialized.

Keywords: Risk Assessment; Qualification; Bioreactors 